\title{
Susanne Brandt*
}

\section{„Wie entsteht eigentlich Zukunft?“}

\author{
Entwicklungsprozesse beim Projekt „Das weiße Blatt“ der Büchereizentrale \\ Schleswig-Holstein
}

https://doi.org/10.1515/bfp-2020-0016

Zusammenfassung: Mit dem Projekt „Das weiße Blatt“ hat die Büchereizentrale Schleswig-Holstein 2018 einen Prozess begonnen, der in besonderer Weise eine lebendige Auseinandersetzung mit Zukunftsfragen anregt und sich an einer Bildung für nachhaltige Entwicklung orientiert. Kennzeichen des Projekts sind die Offenheit für Kooperationen und eine dynamische Fortsetzung, auch über den ursprünglichen Projektzeitraum hinaus, geprägt von einer multimedialen, digitalen wie analogen Vielfalt, die sich bis heute aus den Impulsen des Projekts mit kreativ gestalteten Formen wie E-Book, Film, Podcast, Blog u.v.m. laufend weiterentwickelt. Von elementarer Bedeutung ist dabei außerdem die globale Perspektive, die in verschiedenen internationalen Bezügen des Projekts zum Ausdruck kommt. Mit dem folgenden Bericht wird dieser Entwicklungsprozess von den Grundüberlegungen bis hin zu den vielfältigen Variantenbildungen, die seither aus dem Projekt hervorgegangen sind - auch aktuell unter den besonderen Bedingungen der Pandemie - nachgezeichnet. Ende offen ...

Schlüsselwörter: Nachhaltigkeit; Agenda 2030; Zukunft; Bildung für nachhaltige Entwicklung; frühkindliche Bildung; Kooperationen

\section{"How Does the Future Actually Come About?" The process of development in the project "The blank page" of the library centre Schleswig-Holstein}

Abstract: With the project "The blank page", the library centre of Schleswig-Holstein started a process in 2018 that particularly stimulates a discussion of future issues and that's geared towards education for sustainable development. The characteristic aspects of the project are the openness for cooperation and a dynamic continuation,

Hinweis: Bei dem Beitrag handelt es sich um eine überarbeitete, aktualisierte und stark erweiterte Fassung auf der Basis eines Beitrags, der 2019 im folgenden Sammelband erschienen ist: https:// www.bock-und-herchen.de/components/com_jshopping/files/ demo_products/Hauke_Petra-Oeffentliche_Bibliothek_2030.pdf.

*Kontaktperson: Susanne Brandt, brandt@bz-sh.de even beyond the project period, characterized by a multimedia, digital, and analogue diversity, which continues to evolve from the impulses of the project to this day with creatively designed forms such as e-books, video clip, podcast, blog and much more. The global perspective, which is expressed in various international aspects of the project, is of fundamental importance. The following report traces this development process from the basic considerations to the diverse variants that have emerged from the project since then-also currently under the special conditions of the pandemic. Open end ...

Keywords: Sustainability; SDG; future; education for sustainable development; early childhood education; cooperation

Wer am Schreibtisch spürt, dass es dringend nötig wäre, die Augen zu entspannen, etwas an der erstarrten Haltung zu verändern und tief durchzuatmen, öffnet das Fenster oder tritt vor die Tür, macht vielleicht einen Spaziergang oder arbeitet eine Weile im Garten.

Wer in Bibliotheken nach Visionen des Wandels fragt, weiß sich zugleich eingebunden in institutionelle Strukturen. Bei vielen Planungen, die die Zukunft betreffen, geht es um Prozesse, die messbare Veränderungen erbringen sollen. Es geht um Steigerungs- und Optimierungsbestrebungen, um Innovation und Anpassung an veränderte Gegebenheiten. Das alles soll vorausschauend und kontrolliert geschehen.

Manchmal aber spüren wir es auch hier. Wir brauchen zugleich noch etwas anderes, was sich der genauen Kalkulation entzieht: einen langen Atem, den Blick ins Weite, Begeisterung und frischen Wind.

Also raus aus den gewohnten Räumen - dorthin, wo ein anderer Wind weht: in die Natur, in die Werkstatt oder ins Atelier, wo andere Menschen vielleicht mit ähnlichen Fragen ganz anders umgehen. Oder aus anderer Perspektive etwas entdecken, was wir von dieser Seite so noch nie betrachtet haben. 


\section{Zukunft braucht Inspiration durch Kunst und Kultur}

Was kann passieren, wenn wir uns nicht allein im vertrauten institutionellen Rahmen bewegen? Wir kommen in Austausch, in Resonanz mit einer Umwelt, die nicht allein aus Dingen, Informationen und berechenbaren Faktoren besteht. Und wir spüren vielleicht: Zukunft entsteht nicht allein nach dem Prinzip von Machbarkeit, Verfügbarkeit und Optimierung. Ideen für die Zukunft, die sich früher oder später auch im institutionellen Rahmen zu bewähren haben, sind ebenso angewiesen auf den Anruf und die Inspiration durch Kunst und Kultur, auf die Beziehung zu anderen Menschen und zur Welt - nah und fern - mit ihren brüchigen, widersprüchlichen und erstaunlichen Anteilen, niemals verfügbar und im Detail vorhersehbar.

Aus dem Staunen, aus Begegnungen mit Kunst und Kultur, mit anderen Menschen, ihren Denkweisen und Ideen, schöpfen wir Veränderungsenergie für das, was wir in unserem Bereich bewegen möchten. Natürlich setzen wir dabei auch unsere bewährten Kompetenzen ein, bleiben an Rahmenbedingungen gebunden und werden konkrete Pläne schmieden - aber alles das bliebe eher blass und ohne Strahlkraft, solange es nicht mit der Leidenschaft und Liebe zur Welt betrieben wird, die letztendlich auf Resonanz beruht wie Hartmut Rosa ${ }^{1}$ sie beschreibt.

Denn Zukunftsfähigkeit basiert auf sinnlicher Erkenntnis und der Möglichkeit, Selbstwirksamkeit im Austausch mit anderen zu erfahren - so etwa skizziert es auch Jürgen Manemann, ${ }^{2}$ mit seinen Überlegungen zur Kulturgesellschaft. Für ihn geht es dabei nicht vorrangig um „technische, sondern um kulturelle Herausforderungen, durch die die Frage nach dem Warum ins Zentrum der Debatten gerückt wird. [...] Der Schlüsselbegriff dieser ,Großen Transformation' ist Empowerment: Wir müssen den Mut zur Selbstveränderung aufbringen.“3

1 Vgl. Rosa (2017) http://tagederutopie.org/archiv/tage-der-utopie2017/resonanz.

2 Vgl. Manemann (2017).

3 Manemann (2017) https://fiph.de/veroeffentlichungen/journale/ cover-downloads/fiph_030_RZ_Journal_Ausgabe_29_Schwerpunktth ema_Juergen_Manemann.pdf?m=1570630414\&.

\section{Nachhaltige Entwicklung durch Bibliotheken fördern - aber wie?}

Was bedeutet das nun konkret für die Zukunft von Bibliotheken?

$\mathrm{Zu}$ befragen sind dabei die Nachhaltigkeit bibliothekarischer Arbeit im Blick auf die Zukunftsziele der Agenda 2030, verbunden mit vielfältigen Bildungs- und Teilhabemöglichkeiten von Menschen, die in Bibliotheken nicht allein Informationen suchen, sondern ebenso Gelegenheit zur sinnlichen Erkenntnis in der zwischenmenschlichen Begegnung wie im kreativen oder künstlerischen Erleben.

Bei der Agenda $2030^{4}$ handelt es sich um ein nachhaltiges Zukunftsprogramm mit umfassenden Zielen für die gesamte Welt, durch das bereits in vielen Bereichen des Lebens ein Umdenken und ein verändertes Handeln begonnen hat - und weiterhin geschehen muss. Die Vereinten Nationen haben das Programm 2015 mit Zustimmung von 193 Staaten verabschiedet. Zum Ausdruck gebracht wird damit die gemeinsame Verantwortung für Leben auf diesem Planeten.

17 Ziele mit diversen Unterzielen (international auch SDGs genannt für Sustainable Development Goals) werden mit der Agenda 2030 benannt und überprüft. Im Kern folgen diese Ziele fünf zentralen Botschaften, den sog. ,5 P’s“: für die Bereiche „People“ (Menschen), „Planet“ (Erde), „Prosperity“ (Wohlstand), „Peace“ (Frieden), „Partnership“ (Partnerschaft). ${ }^{5}$

Die Agenda 2030 bildet damit einen komplexen Orientierungsrahmen für nachhaltige Entwicklung zur Umsetzung in der Politik wie zur Suche und Verwirklichung von kreativen Strategien im alltäglichen Handeln.

Bei diesem großen gemeinsamen Anliegen einer nachhaltigen Entwicklung sind Bibliotheken ebenso herausgefordert wie viele andere Akteure des öffentlichen und politischen Lebens.

Nun könnte man auf die Idee kommen, das Kerngeschäft von Bibliotheken einfach unter dem Aspekt der Nachhaltigkeit zu betrachten und für gut zu befinden:

- Bibliotheken schaffen freien Zugang zu Informationen. Davon profitieren (auch) alle, die sich praktisch oder forschend mit Themen der Nachhaltigkeit befassen oder insgesamt die Teilhabe an Bildung suchen.

- Bibliotheken tragen beim Teilen und Verleihen von analogen und digitalen Medien wie auch technischer

4 https://www.bundesregierung.de/breg-de/themen/nachhaltigkeit spolitik/ziele-fuer-eine-nachhaltige-entwicklung-weltweit-355966.

5 https://www.micha-initiative.de/nachhaltigkeitszielesdgs/die-gro sse-transformation-das-sdg-paradigma. 
Ausstattung vermutlich dazu bei, dass weniger Ressourcen verschwendet werden.

- Bibliotheken stellen Raum für Begegnung, Kommunikation und Austausch zur Verfügung - und unterstützen so die Demokratisierung wie auch das soziale $\mathrm{Zu}$ sammenleben in der Kommune.

Das ist ein Anfang. Messbar und vielleicht auch steigerungsfähig sind solche Angebote mittels Ausleihstatistiken, Klicks und Besucherzählern. Aber nachhaltig wirksam? Wir wissen es nicht sicher. Und leider spüren wir es nur selten, solange wir mit kurzfristiger Perspektive allein die Daten und Dinge betrachten. Weitreichendere Erkenntnisse lassen sich vermutlich erst über einen längeren Zeitraum sammeln, wenn Erfahrungen und Entdeckungen aus verschiedenen Perspektiven und Quellen zusammengetragen werden und in einen kooperativ und interdisziplinär gestalteten Transformationsprozess münden.

Vor dieser komplexen Herausforderung stehen nicht allein Bibliotheken. Ich erinnere mich an eine Konferenz zum Thema Nachhaltigkeit in der frühen Bildung, bei der Kitas mehrheitlich davon berichteten, dass dort ein wichtiger Beitrag zur nachhaltigen Entwicklung darin gesehen wird, die Mülltrennung richtig einzuüben. Die Kinder sollen lernen, in welche Tüte welcher Abfall gehört. Dazu wird vielleicht noch die Wasserspartaste an der Toilette erklärt. Ein Anfang auch hier - fokussiert auf Dinge und Verhaltensregeln, die wichtig und richtig sind. Aber welche Chance und Aufmerksamkeit bekommen jene Alltagssituationen, in denen sich Kinder mit Fantasie und eigenen Ideen selbst einbringen können in das Entdecken und Mitgestalten der Welt?

\section{Wissen allein treibt nicht zum nachhaltigkeitsorientierten Handeln an}

Ein Erfahrungsfeld, das nachhaltiges Tun mit Fantasie, Begeisterung und Ideenentwicklung verbindet, ist vor allem in der kulturellen Bildung zu suchen. Diese also gewinnt an Bedeutung, wenn in Kitas, Schulen und Bibliotheken neue Wege einer Bildung für nachhaltige Entwicklung entdeckt und erprobt werden.

Olaf Zimmermann, Geschäftsführer des Deutschen Kulturrates, ${ }^{6}$ beschreibt das, was dabei passieren kann, so:

6 Deutscher Kulturrat (2019).
„In der Kunst und auch in der kulturellen Bildung wollen wir nicht erziehen. Es geht um einen anderen Zugang, den man zu etwas hat, zum Beispiel sehen zu lernen. Wenn ich dieses Fantastische in der Natur sehe, dann liebe ich es auch, dann zerstöre ich es auch nicht. Dann habe ich einen ganz besonderen Blick dafür. Und das ist das, was wir erreichen können und was der Mehrwert kultureller Bildung ist, dass man eine andere Sicht auf die Natur bekommt."7

„Was bewegt Menschen eigentlich zu umweltgerechtem, nachhaltigkeitsorientiertem Handeln?“ fragt auch Heike Molitor, Professorin für Umweltbildung/Bildung für nachhaltige Entwicklung an der Hochschule für nachhaltige Entwicklung Eberswalde: „Es ist eben nicht das Wissen, was mich treibt, sondern es ist ein emotionaler Zugang. Der erste Reflex ist immer: Man muss es den Menschen nur sagen, dann machen sie's. Und genau das - das wissen wir - funktioniert eben nicht; es ist nicht das kognitive Durchdringen. Es braucht einen Perspektivwechsel, und das kann kulturelle Bildung recht gut, mit anderen Methoden und Zugängen als wir das bisher probiert haben." ${ }^{* 8}$

Und Kai Niebert, Präsident des Deutschen Naturschutzrings, ergänzt:

„Worum es doch geht - und das zeichnet auch gute Kultur aus ist, dass sie Menschen erreicht, Menschen berührt, dass sie Menschen ins Nachdenken über sich selber bringt. Ich finde den Begriff ,Bildung für Nachhaltige Entwicklung' einen ganz, ganz unglücklichen Begriff. Bildung findet nie für etwas statt. Wir wollen nicht instrumentalisieren." ${ }^{9}$

Aus der Sicht der Künstlerin Bärbel Rothhaar, Co-Leiterin des Bienen-Kunstprojekts „Moabees“, lässt sich der Gedanke wie folgt weiterführen:

\footnotetext{
„In meiner künstlerischen Arbeit mit Bienen seit 20 Jahren habe ich immer wieder mal festgestellt, dass ich mich fast selber mehr verändere, als dass ich die Welt verändere. Aber vielleicht ist das ja der erste Schritt, auch die Welt zu verändern, dass man bereit ist, sich selbst zu verändern und den Blick zu weiten und andere mitzunehmen auf diesem Weg. [...] Es geht nicht so sehr um reines Wissen, sondern um dieses spielerische Forschen, mit dem wir gern andere anstecken." ${ }^{10}$
}

Doch Vorsicht! Lässt sich aus solchen Erkenntnissen nun schließen, dass sich Kunst und Kultur - auch in Bibliotheken - gezielt einsetzen lassen, um bei der Erfül-

7 Riedel (Moderation) (2018).

8 Riedel (Moderation) (2018).

9 Ebd.

10 Ebd. 
lung der oben erwähnten Nachhaltigkeitsziele im Sinne der Agenda 2030 nachweislich Erfolge zu verzeichnen? Ja und Nein. Denn zugleich gilt: Kunst und Kultur sprechen zunächst für sich und setzen ihre Freiheit aufs Spiel, wenn sie sich politisch vereinnahmen lassen. Jeder Versuch, sie für die Agenda 2030 als ein weltweites politisches Programm $\mathrm{zu}$ instrumentalisieren, stellt sich gegen das verändernde, befreiende und kreative Potential von Kunst und Kultur. Gleichwohl können die Themen und Ziele der Agenda 2030 einen Prozess der künstlerischen Auseinandersetzung anregen. Und die Ausdrucksformen dieser Auseinandersetzung können die Motivation und neue Ideen zur konkreten Mitgestaltung der Gegenwart für eine gute Zukunft beleben und mit wichtigen Impulsen und Energien bereichern.

\section{Weltbilder und Bilderwelten zum Weiterdenken mit Kindern}

All diese Vorüberlegungen spielten eine entscheidende Rolle, als die Büchereizentrale Schleswig-Holstein ab Herbst 2017 konkrete Ideen dazu entwickelte, in welcher Weise sie als zentrale Einrichtung Impulse und Medien zur künstlerischen und kulturellen Auseinandersetzung mit dem Thema Nachhaltigkeit und Agenda 2030 in den Öffentlichen Bibliotheken des Landes einbringen könnte und zwar vor allem im Bereich der frühkindlichen Bildung.

Es sollte dabei um Medien, um neue Kooperationen wie um künstlerische Zugänge zur Inspiration eigener Ideen gehen - und ganz wichtig: Von Anfang an sollten Kinder und Jugendliche Gelegenheit erhalten, sich kreativ und künstlerisch mit ihren persönlichen Erfahrungen, Wünschen und Befürchtungen in dieses Projekt einzubringen. Ihre Fragen und Vorstellungen sollten zum Ausgangspunkt für alle weiteren Überlegungen und Entscheidungen zur Medienauswahl und zur Weiterentwicklung des Projekts werden.

Durch eine Förderung aus dem Fonds Nachhaltigkeitskultur ${ }^{11}$ konnten die Ideen schließlich mit landesweiter Ausstrahlung zur Umsetzung kommen. Der Förderung war ein Ideenwettbewerb vorausgegangen, bei dem die Büchereizentrale mit ihrer Ausgangsidee nominiert und dazu eingeladen wurde, die Idee bei einem „Speed Dating“ in
Berlin verschiedenen Stiftungen und Förderern vorzustellen. Bereits dort ergaben sich vielfältige Austauschmöglichkeiten mit anderen Akteuren und Initiativen, die sich auf unterschiedliche Weise für die Ziele der Agenda 2030 engagieren - und am Ende dann das Angebot, für die weiter ausgearbeitete Idee eine Förderung beim Fonds Nachhaltigkeitskultur zu beantragen.

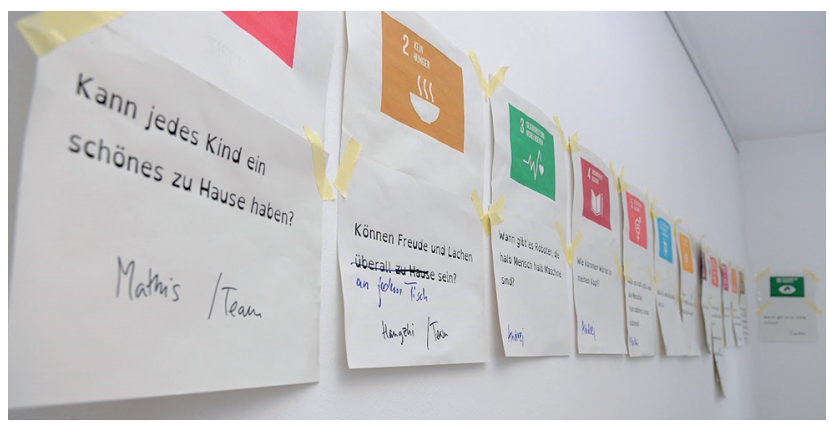

Abb. 1: Agendaziele in der Diskussion

\section{5 „Das weiße Blatt“ - Offenheit und Kreativität bis heute}

Der mit der Idee geborene Projekt-Titel „Das weiße Blatt“12 lässt einerseits an ein Kamishibai als Erzählmedium mit seinen losen Blättern denken, die sich überall direkt und unkompliziert von Kindern und Jugendlichen gestalten lassen. Zugleich steht das „weiße Blatt“ aber auch unabhängig von der jeweils gewählten Vermittlungsform für die Offenheit, mit der die Weltbilder und Bilderwelten von Kindern in das Projekt einbezogen werden sollten - nicht allein beim Erzählen mit Kamishibai, sondern auch bei einem gemeinsam gestalteten Online-Bilderbuch, bei einem Kurzfilm, bei Impulskarten und einem immerwährenden Aktionstage-Kalender, bei Liedern, einem Podcast und einem Faltbüchlein zum Selbstgestalten - um nur einige Facetten zu nennen, die sich aus dem Projekt heraus entwickelt haben. All diese Medienformen lassen Raum als „weißes Blatt“ im symbolischen Sinne - für die Weltbilder und Bilderwelten der Kinder selbst.

Es galt also, ein Projekt zu verwirklichen, das einerseits an gewisse institutionelle Vorgaben gebunden bleibt und einem verbindlichen Plan folgen muss (auch gegenüber dem Fonds Nachhaltigkeitskultur als Geldgeber), an-
11 https://www.nachhaltigkeitsrat.de/projekte/fonds-nachhaltigkei tskultur/.
12 https://www.bz-sh.de/index.php/23-startseite/608-das-weisse-bl att-weltbilder-und-bilderwelten-zum-nachdenken-mit-kindern. 
dererseits aber auch gestalterische Freiheiten zulässt, um die erst im Verlauf entstehenden Ideen der Kinder soweit wie möglich einzubinden, auf unbegrenzte Zeit nutzen und entfalten zu können.

Und ebenso wurde deutlich: Ein solches Vorhaben kann nur in guter Kooperation mit anderen Partnern gelingen, die künstlerische und pädagogische Kompetenzen in diesen Prozess einbringen.

Die Büchereizentrale Schleswig-Holstein fand diese Vielfalt an Kompetenzen beim Kinderliteraturhaus Bücherpiraten e.V. in Lübeck ${ }^{13}$ - und damit zugleich den Kontakt zu zahlreichen Kindern und Jugendlichen wie zu Künstlerinnen und einer Filmemacherin, die mit ihren vielfältigen Erfahrungen die entscheidenden Schritte und Weichenstellungen zu Beginn der Umsetzung im Sommer 2018 mit vorbereiteten, begleiteten und weiterentwickelten.

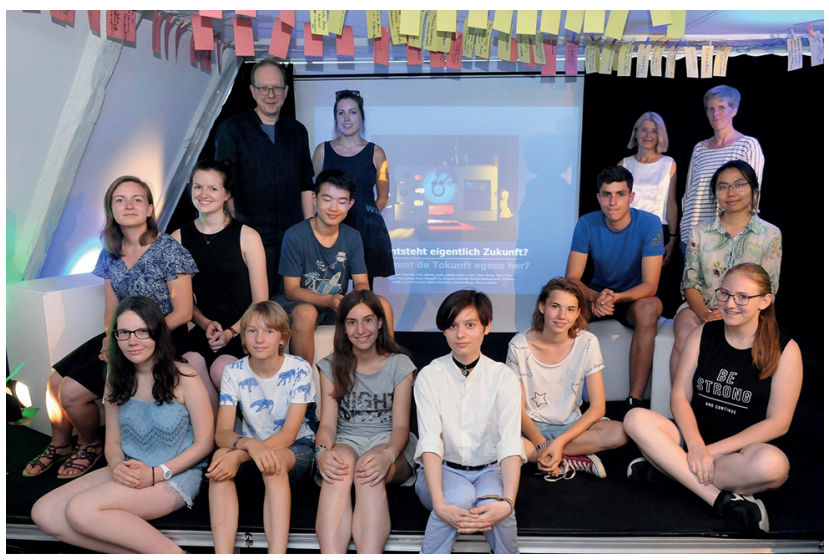

Abb. 2: Werkstatt-Team bei den Bücherpiraten

Ganz im Sinne des kreativen und emanzipativen Ansatzes des Projekts sind bei den Bücherpiraten die Kinder und Jugendlichen aktiv, gestaltend und vor allem auch verantwortlich mit eingebunden in das, was in dem Kinderliteraturhaus in der Lübecker Altstadt mit Sprache, Literatur und Kunst regelmäßig oder auch projektweise geschieht. Sie lernen dabei, gemeinsame Lösungen zu finden, an ihre eigenen Fähigkeiten zu glauben, der Stärke des Teams zu vertrauen und ihre Ziele mit Herz und Kopf zu verfolgen. Das hat sich auch für den Start und Verlauf dieses Kooperationsprojekts als eine wichtige Quelle der Motivation und Weiterentwicklung erwiesen.

\section{Mit dem Staunen fängt es an...}

Mit dem Staunen fängt es an - das Motto der Ferienwerkstatt bei den Bücherpiraten am Anfang der Projektumsetzung war zugleich Programm: Inspiriert von den fünf zentralen Zielbereichen der Agenda 2030 machten sich zunächst Kinder im Vor- und Grundschulalter Gedanken über das, was ihnen in der Gegenwart wie für die Zukunft wichtig ist im Leben - im Realen wie auch im Wunderbaren und Utopischen. Denn jedes Nachdenken über die Zukunft setzt ein Entdecken und Staunen über die Welt, so wie sie gegenwärtig ist, voraus. Und die Lust und Fantasie, über das Vorhandene hinaus zu denken:

- Jeder Mensch ist wertvoll - was bedeutet das? („People“)

- Was können wir zum Frieden beitragen? („Peace“)

- Was brauchen Menschen, um sich wohlzufühlen? („Prosperity“)

- Wie gehen wir mit den Schätzen der Erde um? („Planet")

- Gemeinsam etwas bewegen - wie gelingt das? („Partnership“)

Mehr als 80 Fragen sind den Kindern zu diesen Impulsen in den Sinn gekommen: Dürfen Menschen gegen Menschen sein? Darf man überhaupt noch malen, wenn für Papier Wälder abgeholzt werden? Und ganz direkt: Wie entsteht eigentlich Zukunft? Am Ende ließen sich unter all diesen Fragen Bezüge zu allen 17 Zielen der Agenda 2030 entdecken.

Die gesammelten Kinderfragen wiederum lieferten den Jugendlichen bei den Bücherpiraten nun reichlich Stoff zum Nachdenken und Diskutieren. $\mathrm{Zu}$ einer von den Jugendlichen selbst getroffenen Auswahl aus dieser Fülle von Gedanken über das Leben wurden dann in der sich anschließenden mehrtägigen Atelierzeit Bilder, Collagen und Installationen gestaltet.

Ein erstes Ergebnis dieser grundlegenden Projektphase war schließlich das Online-Bilderbuch „Wie entsteht eigentlich Zukunft“, das bisher in 40 Sprachen übersetzt und online auf dem Portal „Bilingual picture books“14 der Bücherpiraten in Lübeck bereitgestellt worden ist. 


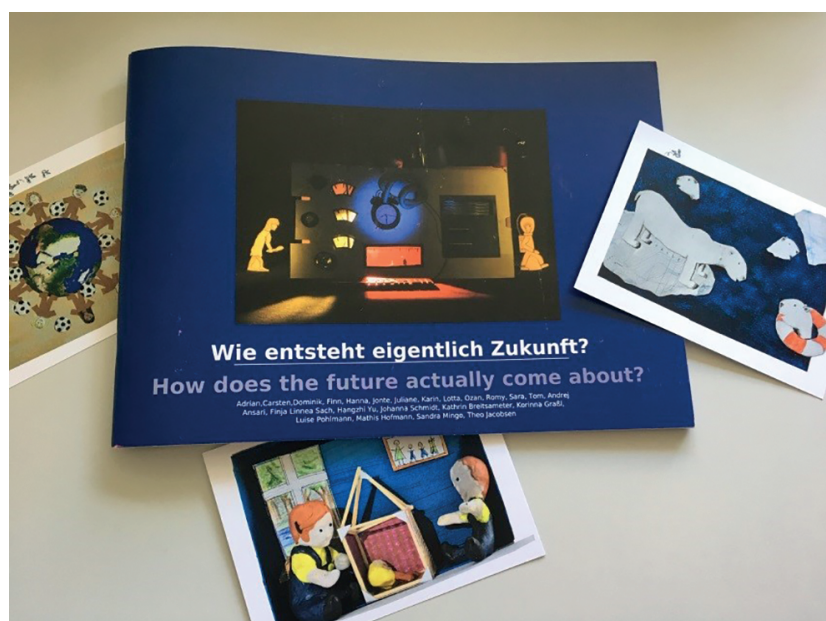

Abb. 3: Buch „Wie entsteht eigentlich Zukunft?“

Die Mehrsprachigkeit und weltweite Verfügbarkeit war den Jugendlichen auch deshalb wichtig, weil die Fragen der Kinder zur Zukunft der Welt nicht nur in Deutschland relevant sind, sondern auch in anderen Regionen der Erde zum Nachdenken anregen können. Vorbereitet und moderiert wurde die Ferienwerkstatt bei den Bücherpiraten e.V. in Lübeck von Martin Gries und Anne Hofmann in Kooperation mit der Büchereizentrale Schleswig-Holstein.

\section{Medienauswahl orientiert sich an den Fragen der Kinder}

Erst nach dieser intensiven inhaltlichen Vorarbeit unter Einbeziehung zahlreicher Kinder und Jugendlicher kamen nun die Bibliotheksmedien ins Spiel: Die von Jugendlichen gestalteten Bilder $\mathrm{zu}$ den Kinderfragen wurden für das Erzählen mit Kamishibai als Bildkarten ausgedruckt und können so in vielfältiger Weise in Bibliotheken, Kitas und Grundschulen zum Einsatz kommen. Als Arbeitshilfe dazu wurde bei der Büchereizentrale Schleswig-Holstein eine Begleitbroschüre erarbeitet. ${ }^{15}$

Eingebettet in eine Rahmengeschichte oder verbunden mit einem passenden Buch können die Bildkarten zunächst erzählend oder inspirierend mit Kamishibai vorgestellt werden. Bei Grundschulkindern ist es ebenso möglich, die Bilder als Impulskarten zu einzelnen Themen zu

15 Diese wie alle anderen Materialien zum Download unter www.na chhaltig-erzaehlen.de. nutzen, die dann von Kindern in Kleingruppen weiter diskutiert werden. Besonders hierbei können die thematisch passenden Bücher aus den geplanten Medienboxen hilfreich zum Einsatz kommen.

Und am Ende der Bilderserie bleibt weiterhin Platz für ein ,weißes Blatt“ - denn weder die Zahl der Fragen noch die Möglichkeiten, darauf Antworten zu finden, sind damit bereits erschöpft oder abgeschlossen. Das wichtigste Ziel des Projekts bleibt die Lust am Weiterfragen!

Eine Liste der rund 50 ausgewählten Buchtitel für Kinder im Vor- und Grundschulalter, die in verschiedenen Bezügen zu den Kinderfragen stehen und den Büchereien ab Frühjahr 2019 als Medienboxen zur Verfügung gestellt werden, ist ebenfalls in der Online-Begleitbroschüre zu finden.

\section{Weiterentwicklung auch in Zukunft - multimedial}

Es gehört zu den besonderen Chancen des Projekts, dass die kreative und multimediale Nutzung und Weiterentwicklung der Materialien wie des Themas insgesamt auch seit Ende der geförderten 12-monatigen Projektzeit eine lebendige Fortsetzung erfahren kann. Möglich ist das durch die stabile Infrastruktur und das gute Netzwerk der Büchereizentrale. So können seither und in Zukunft von hier aus weitere Impulse gegeben, Ideen aus dem Netzwerk geteilt und Interessierte in Schleswig-Holstein und anderswo mit Workshops in der Praxis begleitet und unterstützt werden.

Entsprechend groß ist die Vielfalt an digitalen wie analogen Medien und Vermittlungsformen, die seit Beginn rund um das Projekt entstanden sind.

Die 15 wichtigsten Bausteine mit verschiedenen medialen Formen im Überblick (Stand Frühjahr 2020):

- Online-Bilderbuch in 40 Sprachen (wahlweise als Print-Ausgabe in diversen Sprachkombinationen)

- Ferien-Workshop mit Jugendlichen

- Kamishibai-Bildkartensätze zum Entdecken und Erzählen

- Impulskarten für Diskussionsrunden

- 20 Medienboxen zur Agenda 2030

- Trickfilm mit Musik als Impulsgeber

- Mitmach-Liederheft

- Immerwährender Kalender mit Medien-Tipps und Aktionstagen

- Handreichung für die Praxis

- mobile Workshop-Formate für die Vermittlung im Inund Ausland 
- interaktives Ausstellungskonzept mit Materialien aus dem Projekt, erstellt und umgesetzt in Požega/Serbien $^{16}$

- Podcast der Bücherpiraten, bei dem Kinder zu den Fragen zu Wort kommen ${ }^{17}$

- kreatives Ergänzungsangebot mit dem Schwerpunkt „Kultur \& Natur“ für Familien in der Corona-Zeit: „Vor der Haustür die Welt" ${ }^{\text {18 }}$

- kreative Autoren-Begegnung zum Buch „Wasser ist nass" mit einer Schulklasse in Kiel

- laufend ergänzte Blog-Beiträge für die Praxis ${ }^{19}$

\section{Lebendiger Austausch zu globalen Themen - auch international}

Im Verlauf der bislang zweijährigen Praxis mit dem Projekt ist deutlich geworden: Das bereits vorhandene Material ist auch im internationalen Austausch von großer inspirierender Kraft. So hat z. B. wenige Wochen nach der Ferienwerkstatt eine Bücherei im serbischen Požega auf das OnlineBilderbuch zugegriffen. Dank der dort hinterlegten serbischen Übersetzung konnten sie die Fragen und Bilder bereits Anfang Oktober in ein eigenes Projekt mit Ausstellung zur Agenda 2030 einbinden.

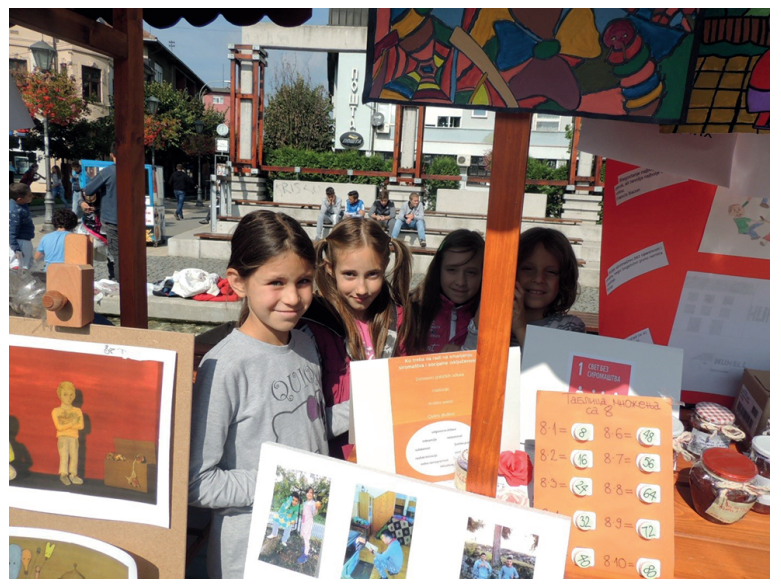

Abb. 4: Umsetzung international in Požega

16 http://www.bz-sh-medienvermittlung.de/fragen-lesen-denken-m alen-singen/.

17 http://www.bz-sh-medienvermittlung.de/hoer-tipp-zum-bilderbu ch-wie-entsteht-eigentlich-zukunft/.

18 http://www.bz-sh-medienvermittlung.de/vor-der-haustuer-die-w elt-umsicht-ueben-und-freiraeume-entdecken/.

19 http://www.bz-sh-medienvermittlung.de/thema/agenda-2030-pr ojekt/.
Einladungen durch das Goethe-Institut nach Kroatien und eine Fortbildungen in Österreich, Veröffentlichungen zum Projekt in den Fachpublikationen dieser Länder und die Teilnahme an internationalen Tagungen zur Agenda 2030 auf Einladung durch den Rat für nachhaltige Entwicklung haben weitere Kontakte mit Akteuren aus aller Welt entstehen lassen, die bis heute andauern.

Aus der Praxis für die Praxis in kleinen wie größeren Bibliotheken sind immer wieder neue Umsetzungsideen entstanden, die dazu taugen, in mehrsprachigen Fassungen den internationalen Austausch zu dem Thema zwischen Büchereien in verschiedenen Ländern weiter anzuregen. Dabei erweist sich die Medienvielfalt mit OnlineBilderbuch, analogen Bildkarten, Kurzfilm, Bücherkisten und Materialien, die wiederum online über www.nachhaltig-erzaehlen.de weit über Schleswig-Holstein hinaus Verbreitung finden, als gute Voraussetzung für eine lebendige Kommunikation und Weiterentwicklung des Themas auf verschiedenen Wegen.

\section{Fazit}

Die Entwicklung und Umsetzung des Projekts haben bereits nach wenigen Monaten gezeigt, dass sich die Wahrnehmung von bibliothekarischem Engagement auf diesem Gebiet vor allem dort verändert hat, wo man die Bedeutung von Bibliotheken im Bereich Bildung für nachhaltige Entwicklung und Agenda 2030 bislang kaum im Blick hatte.

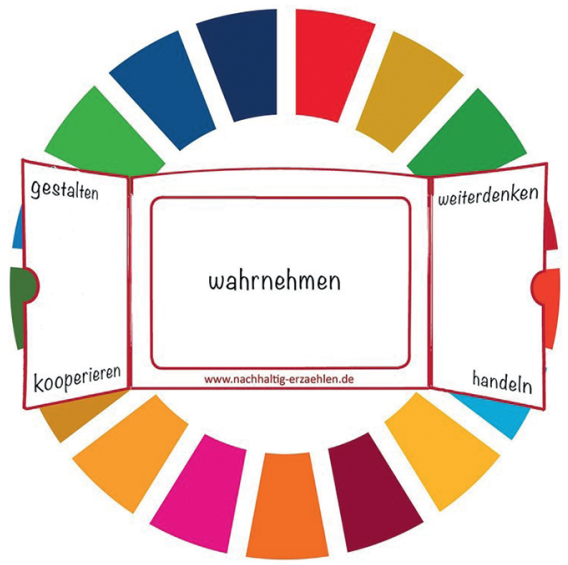

Abb. 5: Wahrnehmung im Zentrum

Davon abgesehen können Bibliotheken durch Projekte wie diese auch noch in anderer Weise für ein zukunftsweisendes Engagement lernen und profitieren:

- Es ist gut, die Kooperation und den Austausch mit Partnern außerhalb des Bibliothekswesens zu intensi- 
vieren und sich dabei gegenseitig mit verschiedenen Kompetenzen zu stärken.

- Es ist gut, sich durch solche Kooperationen auch hinterfragen zu lassen: Ist das, was wir tun und wie wir es bisher getan haben, wirklich nachhaltig im umfassenden Sinne? Brauchen wir mehr Zeit, Ressourcen und Offenheit, um außerbibliothekarische Kontakte zu knüpfen und zu pflegen?

- Es ist gut, auf gesellschaftliche Herausforderungen nicht allein mit „Information“ und „Wissensvermittlung“ zu reagieren, sondern Teilhabe und kulturelle Bildung weit darüber hinaus zu denken und auszugestalten.

Und für alle drei Punkte gilt: Es ist gut, zunächst interessiert zuzuhören, aufmerksam wahrzunehmen, wie und warum sich andere engagieren und lernend im Gespräch zu bleiben mit Akteuren, die vielleicht für ähnliche Anliegen andere Wege gehen. So reifen Ideen, Einsichten und Entscheidungen für neue Möglichkeiten des Miteinanders.

Denn auch für die Zukunft von Bibliotheken gilt immer wieder: Mit dem Staunen fängt es an...

\section{Literaturverzeichnis}

Deutscher Kulturrat (2019): Umsetzung der Agenda 2030 ist eine kulturelle Aufgabe: Positionspapier des Deutschen Kulturrates zur UN-Agenda 2030 für nachhaltige Entwicklung. Verfügbar unter https://www.kulturrat.de/positionen/umsetzung-deragenda-2030-ist-einekulturelle-aufgabe/Deutschland.

Hauke, Petra (Hrsg.) (2019): Öffentliche Bibliothek 2030.

Herausforderungen - Konzepte - Visionen. Bad Honnef: Bock + Herchen, lizenziert nach CC BY-NC-SA 4.0. Verfügbar unter http://www.bock-und-herchen.de/oa/304.

Manemann, J. (2017): Für eine neue Humanökologie. In: fiph-Journal, (29), 14-20. Verfügbar unter https://fiph.de/veroeffentlichun gen/journale/coverdownloads/fiph_030_RZ_Journal_Ausgabe_ 29_Schwerpunktthema_Juergen_Manemann.pdf?m=14926772 15.

Riedel, A. (Moderation) (2018): Kulturelle Bildung und Nachhaltigkeit: Zum Lernen gehören Emotionen und Perspektiven [Radioübertragung]. Berlin: Deutschlandfunk Kultur vom 15. Juni 2018. Verfügbar unter https://www.deutschlandfunkkultur.de/kul turelle-bildung-und-nachhaltigkeitzum-lernen-gehoeren.1083. de.html?dram:article_id=420396.

United Nations (2015): Transforming our world: The 2030 Agenda for Sustainable Development. [New York, NY: United Nations, Division for Sustainable Development]. Verfügbar unter https://sus tainabledevelopment.un.org/content/documents/21252030\%2 0Agenda\%20for\%20Sustainable\%20Development\%20web.pdf.

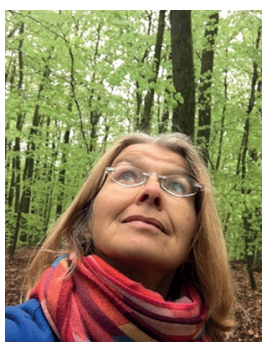

\section{Susanne Brandt}

Büchereizentrale Schleswig-Holstein

Waitzstr. 5

D-24937 Flensburg

brandt@bz-sh.de 